Groups Geom. Dyn. 9 (2015), 55-65

DOI $10.4171 /$ GGD/305
Groups, Geometry, and Dynamics

(C) European Mathematical Society

\title{
Graphs and two-step nilpotent Lie algebras
}

\author{
Meera G. Mainkar
}

\begin{abstract}
We consider a method popular in the literature of associating a two-step nilpotent Lie algebra with a finite simple graph. We prove that the two-step nilpotent Lie algebras associated with two graphs are Lie isomorphic if and only if the graphs from which they arise are isomorphic.
\end{abstract}

Mathematics Subject Classification (2010). 22E25.

Keywords. Nilpotent Lie algebras.

\section{Introduction}

Recently in connection with the study of some very interesting geometrical and dynamical properties of nilmanifolds, the simply connected nilpotent Lie groups or equivalently their Lie algebras have received the attention of many researchers. Among these nilpotent Lie algebras, the two-step ones are the simplest (after the abelian ones) and most widely studied $[1,2,3,4,5,6,10,11,12,13,14,15,17,18$, 19, 20].

Let $k$ be a field with characteristic not equal to 2. We recall that a non-abelian finite dimensional Lie algebra $\mathfrak{n}$ over $k$ is said to be a two-step nilpotent Lie algebra if $[\mathfrak{n},[\mathfrak{n}, \mathfrak{n}]]=\{0\}$. Every two-step nilpotent Lie algebra $\mathfrak{n}$ over $k$ can be realized as a vector space direct sum $V \oplus\left(\bigwedge^{2} V\right) / W$, where $V$ is a finite dimensional $k$-vector space and $W$ is a subspace of the exterior power $\wedge^{2} V$. The Lie bracket structure on $\mathfrak{n}$ is given by

(1) $\left[v_{1}, v_{2}\right]=v_{1} \wedge v_{2} \bmod W$ for $v_{1}, v_{2} \in V$, and

(2) $[x, y]=0$ for $x \in \mathfrak{n}$ and $y \in\left(\bigwedge^{2} V\right) / W$

A combinatorial approach for construction of two-step nilpotent Lie algebras was described in [1]. Subsequently this construction has been used by many authors $[12,13,20,18]$. We recall the construction. Let $(S, E)$ be a finite simple 
graph, where $S$ is the set of vertices and $E$ is the set of edges, where it is assumed that there are no loops and no multiple edges connecting the same pair of vertices. We associate with $(S, E)$ a two-step nilpotent Lie algebra $\mathfrak{n}=\mathfrak{n}(S, E)$ over $k$ in the following way. The underlying vector space of $\mathfrak{n}$ is $V \oplus\left(\bigwedge^{2} V\right) / W$, where $V$ is the $k$-vector space consisting of formal $k$-linear combinations of elements of $S$ (so that $S$ is a basis of $V$ ), and $W$ is the subspace of the exterior power $\wedge^{2} V$ spanned by the vectors $\alpha \wedge \beta$, where $\alpha \beta \notin E$. The Lie bracket structure on $\mathfrak{n}$ is defined as above. We note that the space $\left(\bigwedge^{2} V\right) / W$ has dimension $|E|$. In fact it is spanned by $\{\alpha \wedge \beta \bmod W \mid \alpha \beta \in E\}$. Consequently $\mathfrak{n}(S, E)$ has dimension $|S|+|E|$.

Note that the construction of $\mathfrak{n}(S, E)$ form the graph $(S, E)$ is functorial in the sense that if $f:(S, E) \rightarrow\left(S^{\prime}, E^{\prime}\right)$ is an isomorphism of graphs, then we obtain an isomorphism $f_{*}: \mathfrak{n}(S, E) \rightarrow \mathfrak{n}\left(S^{\prime}, E^{\prime}\right)$ in the natural way: $f_{*}(\alpha)=f(\alpha)$ for each $\alpha \in S$ and for each edge $\alpha \beta \in E, f_{*}(\alpha \wedge \beta)=f(\alpha) \wedge f(\beta)$. It is easy to see that $f_{*}$ extends linearly to a Lie algebra isomorphism from $\mathfrak{n}(S, E)$ to $\mathfrak{n}\left(S^{\prime}, E^{\prime}\right)$.

In this note, we consider the converse question and prove the following:

Theorem 1.1. Let $(S, E)$ and $\left(S^{\prime}, E^{\prime}\right)$ be finite simple graphs. If the two Lie algebras $\mathfrak{n}(S, E)$ and $\mathfrak{n}\left(S^{\prime}, E^{\prime}\right)$ are isomorphic then the graphs $(S, E)$ and $\left(S^{\prime}, E^{\prime}\right)$ are also isomorphic.

This result has already found use in some recent investigations [18, 20]. In [18], the authors provide a method to construct Einstein solvmanifolds by using the Lie algebras $\mathfrak{n}(S, E)$. Using our main theorem, these solvmanifolds are isometric if and only if the graphs are isomorphic, which gives us examples of nonisometric Einstein solvmanifolds.

In [20], the authors construct symplectic two-step nilpotent Lie algebras associated with graphs. In this case our main result implies that there are exactly five non-isomorphic two-step nilpotent Lie algebras of dimension six associated with graphs in the above manner. Indeed, there are five non-isomorphic graphs $(S, E)$ such that $|S|+|E|=6$. Now, $\operatorname{dim} \mathfrak{n}(S, E)=|S|+|E|$. Hence using our Theorem 1.1, there are exactly five non-isomorphic two-step nilpotent Lie algebras of dimension six associated with graphs. This fact was used in [20, Remark 1].

The group of Lie automorphisms of $\mathfrak{n}(S, E)$ was determined in terms of the graph $(S, E)$ in [1]. Also, Anosov and ergodic automorphisms on corresponding nilmanifolds were studied. In [18], explicit examples and non-examples of Einstein solvmanifolds were constructed using the Lie algebras $\mathfrak{n}(S, E)$ (see also [12]). A combinatorial construction of the first and second cohomology groups of $\mathfrak{n}(S, E)$ was given in [20], and was used to construct symplectic and contact nilmanifolds. 
Remark 1.2. Suppose the underlying field $k=\mathbb{R}$. Let $N(S, E)$ denote the unique simply connected nilpotent Lie group corresponding to the Lie algebra $\mathfrak{n}(S, E)$. Then the Lie group exponential map exp: $\mathfrak{n}(S, E) \rightarrow N(S, E)$ is a diffeomorphism (see [21], p. 6). We note that the Baker-Campbell-Hausdorff formula ([23], p. 119)) gives the multiplication law in $N(S, E)$ as follows:

$$
\exp (x) \exp (y)=\exp \left(x+y+\frac{1}{2}[x, y]\right)
$$

for all $x, y \in \mathfrak{n}(S, E)$.

More precisely, we can realize $N(S, E)$ as $\mathfrak{n}(S, E)$ (via the exponential map) with the multiplication defined by

$$
\left(v_{1}, x_{1}\right) \cdot\left(v_{2}, x_{2}\right)=\left(v_{1}+v_{2}, x_{1}+x_{2}+\frac{1}{2}\left[v_{1}, v_{2}\right]\right)
$$

for all $v_{1}, v_{2} \in V$ and $x_{1}, x_{2} \in\left(\bigwedge^{2} V\right) / W$.

Then our Theorem 1.1 implies that the simply connected Lie groups $N(S, E)$ and $N\left(S^{\prime}, E^{\prime}\right)$ are Lie isomorphic if and only if the graphs $(S, E)$ and $\left(S^{\prime}, E^{\prime}\right)$ are isomorphic. This can been seen by using the fact that the simply connected Lie groups are Lie isomorphic if and only if their Lie algebras are Lie isomorphic (see [24], p. 101).

Remark 1.3. There have been some other constructions of algebraic structures associated with a simple graph. Some, though not all of these are related with the construction considered here. In [16], the authors consider the $K$-algebra associated with a simple graph $(S, E)$ over a field $K$ generated by the set of vertices $S$ and with relations $\alpha \beta=\beta \alpha$ if and only if $\alpha \beta \notin E$. They proved that these $K$-algebras are isomorphic if and only the corresponding graphs are isomorphic. This result was further used to prove an analogous result for graph groups in [7]. In [7], the author considers the group associated with graph $(S, E)$ which is defined as the group generated by the set $S$ and with relations $\alpha \beta=\beta \alpha$ if and only if $\alpha \beta \in E$. Here we remark that the simply connected nilpotent Lie group $N(S, E)$ (as in Remark 1.2) is not finitely generated.

Later in [8], the authors introduce the free partially commutative Lie algebra $\mathfrak{l}(S, E)$ associated with $(S, E)$ which is the quotient of the free Lie algebra on the set $S$ modulo the Lie ideal generated by $\{[\alpha, \beta] \mid \alpha \beta \notin E\}$. In fact, the authors study these structures in more generality but we will not go into the details here. Following $[7,9,16]$, it can be shown that these free partially commutative Lie algebras are Lie isomorphic if and only if the corresponding graphs are isomorphic (see Theorem 1 in [7] for example). Here we note that the quotient of $\mathfrak{l}(S, E)$ 
by the Lie ideal $\mathfrak{l}^{3}$ generated by the set $\{[x,[y, z]] \mid x, y, z \in \mathfrak{l}(S, E)\}$, is Lie isomorphic to our two-step nilpotent Lie algebra $\mathfrak{n}(S, E)$. The isomorphism result for the free partially commutative Lie algebras can be recaptured by using our Theorem 1.1 (if the characteristic of the field $K$ is not equal to 2) as follows. Let $\mathfrak{l}=\mathfrak{l}(S, E)$ and $\mathfrak{l}^{\prime}=\mathfrak{l}\left(S^{\prime}, E^{\prime}\right)$ be Lie isomorphic free partially commutative Lie algebras associated with graphs $(S, E)$ and $\left(S^{\prime}, E^{\prime}\right)$ respectively. Then the quotient Lie algebras $\mathfrak{l} / \mathfrak{l}^{3}$ and $\mathfrak{l}^{\prime} / \mathfrak{l}^{\prime 3}$ are Lie isomorphic. That means the two-step nilpotent Lie algebras $\mathfrak{n}(S, E)$ and $\mathfrak{n}\left(S^{\prime}, E^{\prime}\right)$ are isomorphic and our Theorem 1.1 implies that the graphs $(S, E)$ and $\left(S^{\prime}, E^{\prime}\right)$ are isomorphic.

\section{Some Preliminaries}

Before we prove Theorem 1.1, we need a few facts regarding the structure of the automorphism group of $\mathfrak{n}=\mathfrak{n}(S, E)$. We denote by Aut(n) the group of Lie automorphisms of $\mathfrak{n}$ and $T$ the set of automorphisms $\tau \in \operatorname{Aut}(\mathfrak{n})$ such that $\tau(V)=$ $V$, where we recall that $V$ is the $k$-vector space with basis $S$, and $\mathfrak{n}=V \oplus$ $\left(\bigwedge^{2} V\right) / W$. We denote by $G$ the subgroup of $\operatorname{GL}(V)$ consisting of the linear automorphisms $\left.\tau\right|_{V}$, where $\tau \in T$.

Next we see that the group $G$ consists of $g \in \operatorname{GL}(V)$ such that the subspace $W \subseteq \wedge^{2} V$ is invariant under the induced natural action $\wedge^{2} g$ of $g$ on $\bigwedge^{2} V$. Indeed, if $g \in G$ (i.e. if $g=\left.\tau\right|_{V}$ for some $\tau \in T$ ) and if $\alpha \beta \notin E$, then

$$
\begin{aligned}
g(\alpha) \wedge g(\beta) \bmod W & =[\tau(\alpha), \tau(\beta)] \\
& =\tau[\alpha, \beta] \\
& =0,
\end{aligned}
$$

since $\tau$ is an automorphism of $\mathfrak{n}$ and $[\alpha, \beta]=0$ in $\mathfrak{n}$. Conversely if $g \in \operatorname{GL}(V)$ such that $g(\alpha) \wedge g(\beta) \in W$ for all $\alpha \beta \notin E$, then $\tau=g \oplus \wedge^{2} g$ defines a Lie automorphism of $\mathfrak{n}$ such that $\tau(V)=V$ and hence $g \in G$. Since the condition that an element of $G$ stabilizes $W$ under the natural action on $\bigwedge^{2} V$ is represented by polynomial equations, we have therefore the following:

Lemma 2.1. $G$ is an algebraic group.

In fact, for any two-step nilpotent Lie algebra $\mathfrak{m}=V \oplus\left(\bigwedge^{2} V\right) / W$ one can analogously define the subgroup $G$ of $\mathrm{GL}(V)$ consisting of the restrictions of automorphisms of $\mathfrak{m}$ fixing $V$. A similar argument shows that $G$ is an algebraic group. However our next lemma holds only for two-step nilpotent Lie algebras arising from graphs. We prove that all linear automorphisms of $V$ which can be 
represented as diagonal matrices with respect to the basis $S$ can be extended to Lie automorphisms of $\mathfrak{n}(S, E)$.

Lemma 2.2. Let $D_{S}$ denote the subgroup of $\mathrm{GL}(V)$ consisting of all elements which can be represented as diagonal matrices with respect to the basis $S$ of $V$. Then $D_{S} \subseteq G$.

Proof. We recall that $G$ is a subgroup of $\mathrm{GL}(V)$ consisting of those linear automorphisms of $V$ whose induced action on $\wedge^{2} V$ leaves the subspace $W$ invariant, where $W \subseteq \wedge^{2} V$ spanned by elements $\alpha \wedge \beta$ such that $\alpha \beta \notin E$. Let $d \in D_{S}$, say $d(\alpha)=d_{\alpha} \alpha$ for some nonzero $d_{\alpha}$ 's in $k$ and for all $\alpha \in S$. Then if $\alpha \beta \notin E$, we have $d(\alpha) \wedge d(\beta)=d_{\alpha} d_{\beta}(\alpha \wedge \beta) \in W$. Hence $d \in G$ and $D_{S} \subseteq G$.

\section{Proof of Theorem 1.1}

Let $\bar{k}$ denote the algebraic closure of $k$. First we note that if $F$ is an isomorphism from the Lie algebra $\mathfrak{n}=\mathfrak{n}(S, E)$ to $\mathfrak{n}^{\prime}=\mathfrak{n}\left(S^{\prime}, E^{\prime}\right)$, then $\bar{F}=F \otimes_{k} \operatorname{id}_{\bar{k}}$ is an isomorphism of $\bar{k}$-Lie algebras $\overline{\mathfrak{n}}=\mathfrak{n} \otimes_{k} \bar{k}$ and $\overline{\mathfrak{n}^{\prime}}=\mathfrak{n}^{\prime} \otimes_{k} \bar{k}$. To see this, recall that the Lie bracket in $\overline{\mathfrak{n}}$ is defined by

$$
[x \otimes a, y \otimes b]=[x, y] \otimes a b \quad \text { for all } x, y \in \mathfrak{n} \text { and } a, b \in \bar{k} .
$$

Also $\bar{F}$ is defined by

$$
\bar{F}\left(\sum_{i} x_{i} \otimes a_{i}\right)=\sum_{i} F\left(x_{i}\right) \otimes a_{i} \quad \text { for all } x_{i} \in \mathfrak{n} \text { and } a_{i} \in \bar{k} .
$$

Then $\bar{F}$ is a $\bar{k}$-vector space isomorphism which follows from the fact that $F$ is a $k$-vector space isomorphism. Furthermore, for $x, y \in \mathfrak{n}$ and $a, b \in \bar{k}$ we have

$$
\begin{aligned}
\bar{F}[x \otimes a, y \otimes b] & =\bar{F}([x, y] \otimes a b) \\
& =(F[x, y] \otimes a b) \\
& =[F(x), F(x)] \otimes a b \text { since } F \text { is a Lie algebra isomorphism. } \\
& =[F(x) \otimes a, F(x) \otimes b]=[\bar{F}(x \otimes a), \bar{F}(y \otimes b)] .
\end{aligned}
$$

Now without loss of generality we can assume that the field $k$ is algebraically closed. Indeed if $k$ is not already algebraically closed, and $F$ is an isomorphism from the Lie algebra $\mathfrak{n}=\mathfrak{n}(S, E)$ to $\mathfrak{n}^{\prime}=\mathfrak{n}\left(S^{\prime}, E^{\prime}\right)$, we can replace $\mathfrak{n}$ by $\overline{\mathfrak{n}}, \mathfrak{n}^{\prime}$ by $\overline{\mathfrak{n}^{\prime}}$ and $F$ by $\bar{F}$. Then the new $F$ is an isomorphism of $\bar{k}$-Lie algebras $\mathfrak{n}$ and $\mathfrak{n}^{\prime}$ as 
discussed above. We need to show that the graphs $(S, E)$ and $\left(S^{\prime}, E^{\prime}\right)$ defining the Lie algebras $\mathfrak{n}$ and $\mathfrak{n}^{\prime}$ are isomorphic.

We recall that

$$
\mathfrak{n}=V \oplus\left(\bigwedge^{2} V\right) / W
$$

where $V$ is the $k$-vector space with basis $S$ and $W \subset \bigwedge^{2} V$ is as defined in the previous section. Similarly

$$
\mathfrak{n}^{\prime}=V^{\prime} \oplus\left(\bigwedge^{2} V^{\prime}\right) / W^{\prime}
$$

where $V^{\prime}$ is the $k$-vector space with basis $S^{\prime}$.

The proof will be in three steps. First we construct a new graph $\left(S^{\prime \prime}, E^{\prime \prime}\right)$ isomorphic to the graph $(S, E)$. The set of vertices $S^{\prime \prime}$ will be a basis of the vector space $V^{\prime}$. Then in the next step we look a group $D_{S^{\prime \prime}}$ analogous to the group $D_{S}$ considered in Lemma 2.2. The theorem will follow from the relation between $D_{S^{\prime \prime}}$ and $D_{S^{\prime}}$ to be explained in the third step.

We begin by constructing a new graph $\left(S^{\prime \prime}, E^{\prime \prime}\right)$ isomorphic to the graph $(S, E)$ in the following way. Recall that $V^{\prime}$ denote the $k$-vector space with $S^{\prime}$ as basis and let $W^{\prime}$ denote the subspace of $\wedge^{2} V^{\prime}$ spanned by the wedge products $\alpha \wedge \beta$, where $\alpha$ and $\beta$ are vertices in $S^{\prime}$ not connected by an edge in $E^{\prime}$. Let $\pi: \mathfrak{n}^{\prime} \rightarrow V^{\prime}$ denote the canonical linear projection with respect to the decomposition $\mathfrak{n}^{\prime}=V^{\prime} \oplus$ $\left(\bigwedge^{2} V^{\prime}\right) / W^{\prime}$. We define $S^{\prime \prime}$ denote the subset of $V^{\prime}$ given by $\{\pi(F(\alpha)) \mid \alpha \in S\}$ and $E^{\prime \prime}$ denote the set of unordered pairs $\pi(F(\alpha)) \pi(F(\beta))$ such that $\alpha \beta \in E$, where, recall that $F$ is the isomorphism given between $\mathfrak{n}$ and $\mathfrak{n}^{\prime}$.

It follows that $S^{\prime \prime}$ is a basis of $V^{\prime}$. Indeed, if $\sum_{i=1}^{n} a_{i} \pi\left(F\left(\alpha_{i}\right)\right)=0$ for $a_{i}$ 's $\in \mathbb{R}$ and $\alpha_{i}$ 's $\in S$, then $F\left(\sum_{i=1}^{n} a_{i} \alpha_{i}\right) \in\left[\mathfrak{n}^{\prime}, \mathfrak{n}^{\prime}\right]$. Since $F$ is a Lie algebra isomorphism, we can see that $\sum_{i=1}^{n} a_{i} \alpha_{i} \in[\mathfrak{n}, \mathfrak{n}]$. On the other hand each $\alpha_{i}$ is in $S$ so $\sum_{i=1}^{n} a_{i} \alpha_{i} \in V \cap[\mathfrak{n}, \mathfrak{n}]$, so that $\sum_{i=1}^{n} a_{i} \alpha_{i}=0$. By the linear independence of $S$, each $a_{i}=0$. Now the derived algebras $[\mathfrak{n}, \mathfrak{n}]$ and $\left[\mathfrak{n}^{\prime}, \mathfrak{n}^{\prime}\right]$ have the same dimension since $\mathfrak{n}$ and $\mathfrak{n}^{\prime}$ are isomorphic. Hence $|E|=\left|E^{\prime}\right|$ since $|E|=\operatorname{dim}[\mathfrak{n}, \mathfrak{n}]$ and $\left|E^{\prime}\right|=\operatorname{dim}\left[\mathfrak{n}^{\prime}, \mathfrak{n}^{\prime}\right]$. Hence $|S|=\left|S^{\prime}\right|$ because $\mathfrak{n}$ is of dimension $|S|+|E|$ and $\mathfrak{n}^{\prime}$ is of dimension $\left|S^{\prime}\right|+\left|E^{\prime}\right|$, and $\mathfrak{n}$ and $\mathfrak{n}^{\prime}$ are isomorphic. Thus the sets $S^{\prime}$ and $S^{\prime \prime}$ have exactly the same number of elements and hence $S^{\prime \prime}$ is a basis of $V^{\prime}$ since $S^{\prime}$ is a basis of $V^{\prime}$.

Since the graph $\left(S^{\prime \prime}, E^{\prime \prime}\right)$ as constructed above is isomorphic to the graph $(S, E)$, to prove the theorem, it is enough to show that there exists an isomorphism of graphs $f:\left(S^{\prime}, E^{\prime}\right) \rightarrow\left(S^{\prime \prime}, E^{\prime \prime}\right)$. 
Now as in Lemma 2.2, $D_{S^{\prime}}$ denote the subgroup of GL $\left(V^{\prime}\right)$ consisting of all elements which can be represented as diagonal matrices with respect to the basis $S^{\prime}$ of $V^{\prime}$. By Lemma 2.2, we have $D_{S^{\prime}} \subset G^{\prime}$. Similarly let $D_{S^{\prime \prime}}$ denote the subgroup of $\mathrm{GL}\left(V^{\prime}\right)$ consisting of all elements which can be represented as diagonal matrices with respect to the basis $S^{\prime \prime}=\{\pi(F(\alpha)) \mid \alpha \in S\}$ of $V^{\prime}$.

We claim that $D_{S^{\prime \prime}} \subset G^{\prime}$ as well. To see this, let $d \in D_{S^{\prime \prime}}$ and $d(\pi(F(\alpha))=$ $d_{\alpha} \pi\left(F(\alpha)\right.$ for all $\alpha \in S$ and for some nonzero $d_{\alpha}$ 's in $k$. It suffices to show that if $\gamma$ and $\delta$ are vertices in $S^{\prime}$ not connected by an edge in $E^{\prime}$, then $d(\gamma) \wedge d(\delta) \in W^{\prime}$ (i.e. $[d(\gamma), d(\delta)]=0$ in $\mathfrak{n}^{\prime}$.) Now since $\gamma, \delta \in V^{\prime}$ and $S^{\prime \prime}$ is a basis for $V^{\prime}$, we represent

$$
\gamma=\sum_{\alpha \in S} a_{\alpha} \pi(F(\alpha)) \quad \text { and } \quad \delta=\sum_{\beta \in S} b_{\beta} \pi(F(\beta)),
$$

where each $a_{\alpha}, b_{\beta}$ is in $k$. Since $[\gamma, \delta]=0$, we have

$$
\left[\pi\left(F\left(\sum_{\alpha \in S} a_{\alpha} \alpha\right)\right), \pi\left(F\left(\sum_{\beta \in S} b_{\beta} \beta\right)\right)\right]=0 .
$$

This means that

$$
\left[F\left(\sum_{\alpha \in S} a_{\alpha} \alpha\right), F\left(\sum_{\beta \in S} b_{\beta} \beta\right)\right]=0
$$

because in $\mathfrak{n}^{\prime},\left[v+w, v^{\prime}+w^{\prime}\right]=0$ if and only if $\left[v, v^{\prime}\right]=0$ for $v, v^{\prime} \in V^{\prime}$ and $w, w^{\prime} \in\left(\bigwedge^{2} V^{\prime}\right) / W^{\prime}$. Hence

$$
\left[\sum_{\alpha \in S} a_{\alpha} \alpha, \sum_{\beta \in S} b_{\beta} \beta\right]=0
$$

in $\mathfrak{n}$ since $F$ is an isomorphism. Now we define $\sigma$, an element of GL $(V)$ by $\sigma(\alpha)=d_{\alpha} \alpha$ for each $\alpha \in S$. Then $\sigma \in D_{S}$, where $D_{S}$ is the subgroup of $\operatorname{GL}(V)$ consisting of diagonal automorphisms of $V$ with respect to $S$ and hence $\sigma \in G$ by Lemma 2.2. So

$$
\left[\sum_{\alpha \in S} a_{\alpha} \sigma(\alpha), \sum_{\beta \in S} b_{\beta} \sigma(\beta)\right]=0
$$

in $\mathfrak{n}$ which means that

$$
\left[\sum_{\alpha \in S} a_{\alpha} d_{\alpha} \alpha, \sum_{\beta \in S} b_{\beta} d_{\beta} \beta\right]=0 .
$$

Since $F$ is an isomorphism,

$$
\left[\sum_{\alpha \in S} a_{\alpha} d_{\alpha} F(\alpha), \sum_{\beta \in S} b_{\beta} d_{\beta} F(\beta)\right]=0
$$


in $\mathfrak{n}^{\prime}$ and hence

$$
\left[\sum_{\alpha \in S} a_{\alpha} d_{\alpha} \pi(F(\alpha)), \sum_{\beta \in S} b_{\beta} d_{\beta} \pi(F(\beta))\right]=0 .
$$

Thus $[d(\gamma), d(\delta)]=0$ in $\mathfrak{n}^{\prime}$ as we wanted to show.

By Lemma 2.1, $G^{\prime}$ is an algebraic group. Moreover, $D_{S^{\prime}}$ and $D_{S^{\prime \prime}}$ are maximal tori in the connected component in Zariski topology of identity of $G^{\prime}$. But maximal tori in a connected algebraic group over an algebraically closed field (such as our $k$ ) are conjugate (see [22], p. 108), and hence there exists $g \in G^{\prime}$ such that

$$
D_{S^{\prime}}=g\left(D_{S^{\prime \prime}}\right) g^{-1} \text {. }
$$

To define an isomorphism of graphs $f: S^{\prime} \rightarrow S^{\prime \prime}$, we construct a special element of $D_{S^{\prime}}$. For each $\alpha \in S^{\prime}$, choose a nonzero $d_{\alpha}^{\prime} \in k$ such that unless the set $\{\alpha, \beta\}$ equals the set $\{\gamma, \delta\}$ we have $d_{\alpha}^{\prime} d_{\beta}^{\prime} \neq d_{\gamma}^{\prime} d_{\delta}^{\prime}$. Since by hypothesis the field $k$ is algebraically closed, it is in particular infinite, and such $d_{\alpha}$ 's may be chosen. Define $d^{\prime} \in D_{S^{\prime}}$ be the element whose matrix representation with respect to $S^{\prime}$ given by $d^{\prime}(\alpha)=d_{\alpha}^{\prime} \alpha$. As note above, there exists $d^{\prime \prime} \in D_{S^{\prime \prime}}$ such that

$$
d^{\prime}=g d^{\prime \prime} g^{-1}
$$

Since $d^{\prime}$ and $d^{\prime \prime}$ are similar, they have the same eigenvalues. Further since $d^{\prime}$ is diagonal with respect to $S^{\prime}$ and $d^{\prime \prime}$ is diagonal with respect to $S^{\prime \prime}$, it follows that these diagonal entries are the same up to a permutation. Hence there exists a bijection $f$ of $S^{\prime}$ with $S^{\prime \prime}$ such that

$$
d^{\prime \prime}(f(\alpha))=d_{\alpha}^{\prime} f(\alpha) \quad \text { for all } \alpha \in S^{\prime}
$$

We claim that $f$ is in fact an isomorphism of the graph $\left(S^{\prime}, E^{\prime}\right)$ with the graph $\left(S^{\prime \prime}, E^{\prime \prime}\right)$. Establishing this claim will conclude the proof of our theorem.

It suffices now to show that $\alpha \beta \in E^{\prime}$ if and only if $f(\alpha) f(\beta) \in E^{\prime \prime}$. Now $\alpha \beta \in E^{\prime}$ if and only if $[\alpha, \beta] \neq 0$ in $\mathfrak{n}^{\prime}$. Also $f(\alpha) f(\beta) \in E^{\prime \prime}$ if and only if $[f(\alpha), f(\beta)] \neq 0$ in $\mathfrak{n}^{\prime}$. This follows from the definition of $E^{\prime \prime}$ and using that $F$ is an isomorphism. Indeed $f(\alpha)=\pi\left(F\left(\alpha^{\prime}\right)\right)$ and $f(\beta)=\pi\left(F\left(\beta^{\prime}\right)\right)$ for some $\alpha^{\prime}, \beta^{\prime} \in S$. Hence $f(\alpha) f(\beta) \in E^{\prime \prime}$ if and only if $\alpha^{\prime} \beta^{\prime} \in E$. Since $F$ is an isomorphism, $\alpha^{\prime} \beta^{\prime} \in E$ if and only if $\left[F\left(\alpha^{\prime}\right), F\left(\beta^{\prime}\right)\right] \neq 0$ in $\mathfrak{n}^{\prime}$ i.e. $\left[\pi\left(F\left(\alpha^{\prime}\right)\right), \pi\left(F\left(\beta^{\prime}\right)\right)\right] \neq 0$ in $\mathfrak{n}^{\prime}$.

We will show that for $\alpha, \beta \in S^{\prime},[\alpha, \beta] \neq 0$ if and only if $[f(\alpha), f(\beta)] \neq 0$. Since the diagonal entries of the chosen element $d^{\prime} \in D_{S^{\prime}}$ (defined as above) are such that the pairwise products of the diagonal entries are distinct, $d_{\alpha}^{\prime} d_{\beta}^{\prime}$ is an eigenvalue of an extended automorphism $d^{\prime}$ of $\mathfrak{n}^{\prime}$ if and only if $[\alpha, \beta] \neq 0$. 
Similarly $d_{\alpha}^{\prime} d_{\beta}^{\prime}$ is an eigenvalue of an extended automorphism $d^{\prime \prime}$ of $\mathfrak{n}^{\prime}$ if and only if $[f(\alpha), f(\beta)] \neq 0$. Now we note that

$$
\left[g^{-1}\left(d^{\prime}(\alpha)\right), g^{-1}\left(d^{\prime}(\beta)\right)\right]=d_{\alpha}^{\prime} d_{\beta}^{\prime}\left[g^{-1}(\alpha), g^{-1}(\beta)\right] .
$$

On the other hand, we have

$$
\left[g^{-1}\left(d^{\prime}(\alpha)\right), g^{-1}\left(d\left({ }^{\prime} \beta\right)\right)\right]=d^{\prime \prime}\left[g^{-1}(\alpha), g^{-1}(\beta)\right], \quad \text { since } g^{-1} d^{\prime}=d^{\prime \prime} g^{-1} .
$$

Since $g \in G^{\prime},\left[g^{-1}(\alpha), g^{-1}(\beta)\right] \neq 0$ if and only if $[\alpha, \beta] \neq 0$. Hence $d_{\alpha}^{\prime} d_{\beta}^{\prime}$ is an eigenvalue of $d^{\prime \prime}$ if and only if $d_{\alpha}^{\prime} d_{\beta}^{\prime}$ is an eigenvalue of $d^{\prime \prime}$. Thus $\alpha \beta \in E^{\prime}$ if and only if $f(\alpha) f(\beta) \in E^{\prime \prime}$. Hence $f:\left(S^{\prime}, E^{\prime}\right) \rightarrow\left(S^{\prime \prime}, E^{\prime \prime}\right)$ is an isomorphism of graphs, this complete the proof of Theorem 1.1.

Acknowledgments. The author was supported by the Central Michigan University ORSP Early Career Investigator Grant C61940.

The author is thankful to Professor Jorge Lauret for raising this question, Professor S. G. Dani for his help, Dr. Debraj Chakrabarti for help with the final version and the referee for helpful suggestions and pointing out the references $[7,8,9,16]$.

\section{References}

[1] S. G. Dani and M. G. Mainkar, Anosov automorphisms on compact nilmanifolds associated with graphs. Trans. Amer. Math. Soc. 357 (2005), 2235-2251. Zbl 1061.22008 MR 2140439

[2] R. DeCoste, L. DeMeyer, and M. Mast, Characterizations of Heisenberg-like Lie algebras. J. Lie Theory 21 (2011), no. 3, 711-727. Zbl 1222.53055 MR 2858081

[3] K. Dekimpe, Hyperbolic automorphisms and Anosov diffeomorphisms on nilmanifolds. Trans. Amer. Math. Soc. 353 (2001), no. 7, 2859-2877. Zbl 0990.37022 MR 1828476

[4] K. Dekimpe and S. Deschamps, Anosov diffeomorphisms on a class of 2-step nilmanifolds. Glasg. Math. J. 45 (2003), no. 2, 269-280. Zbl 1047.37022 MR 1997705

[5] K. Dekimpe and K. Verheyen, Anosov diffeomorphisms on nilmanifolds modelled on a free nilpotent Lie group. Dyn. Syst. 24 (2009), no. 1, 117-121. Zbl 1160.37008 MR 2548819

[6] L. DeMeyer, Closed geodesics in compact nilmanifolds. Manuscripta Math. 105 (2001), no. 3, 28-310. Zbl 1076.53049 MR 1856612

[7] C. Droms, Isomorphisms of graph groups. Proc. Amer. Math. Soc. 100 (1987), no. 3, 407-408. Zbl 0619.20015 MR 0891135

[8] G. Duchamps and D. Krob, Free partially commutative structures. J. Algebra 156 (1993), no. 2, 318-361. Zbl 0854.20065 MR 1216472 
[9] G. Duchamps and D. Krob, The lower central series of the free partially commutative group. Semigroup Forum 45 (1992), no. 3, 385-394. Zbl 0814.20025 MR 1179860

[10] P. Eberlein, Geometry of 2-step nilpotent groups with a left invariant metric. Ann. Sci. École Norm. Sup. (4) 27 (1994), no. 5, 611-660. Zbl 0820.53047 MR 1296558

[11] P. Eberlein, Geometry of 2-step nilpotent Lie groups. Modern dynamical systems and applications. In M. Brin, B. Hasselblatt and Ya. Pesin (eds.). Modern dynamical systems and applications. Cambridge University Press, Cambridge, 2004, 67-101. Zbl 1154.22009 Zbl 1051.00012 (collection) MR 2090766 MR 2090761 (collection)

[12] H.-R. Fanaï, Einstein solvmanifolds and graphs. C. R. Acad. Sci. Paris 344 (2007), 37-39. Zbl 1113.53029 MR 2286585

[13] H.-R. Fanaï, Two-step nilpotent Lie algebras attached to graphs. Int. Math. Forum 4 (2009), no. 41-44, 2143-2148. Zbl 1202.53051 MR 2563387

[14] C. Gordon and Y. Mao, Geodesic conjugacies of two-step nilmanifolds. Michigan Math. J. 45 (1998), no. 3, 45-481. Zbl 0976.53090 MR 1653247

[15] R. Gornet and M. Mast, The minimal marked length spectrum of Riemannian twostep nilmanifolds. Michigan Math. J. 52 (2004), no. 3, 683-716. Zbl 1086.53061 MR 2097405

[16] K. H. Kim, L. Maker-Limanov, J. Neggers, and E. W. Roush, Graph algebras. J. Algebra 64 (1980), no. 1, 46-51. Zbl 431.05023 MR 0575780

[17] J. Lauret and C. Will, Nilmanifolds of dimension $\leq 8$ admitting Anosov diffeomorphisms. Trans. Amer. Math. Soc. 361 (2009), no. 5, 2377-2395. Zbl 1165.37009 MR 2471923

[18] J. Lauret and C. Will, Einstein solvmanifolds: existence and non-existence questions. Math. Ann. 350 (2011), no. 1, 199-225. Zbl 1222.53048 MR 2785768

[19] T. Payne, Anosov automorphisms of nilpotent Lie algebras. J. Mod. Dyn. 3 (2009), no. 1, 121-158. Zbl 1188.37031 MR 2481335

[20] H. Pouseele and P. Tirao, Compact symplectic nilmanifolds associated with graphs. J. Pure Appl. Algebra 213 (2009), 1788-1794. Zbl 1170.53062 MR 2518177

[21] M. S. Raghunathan, Discrete subgroups of Lie groups. Ergebnisse der Mathematik und ihrer Grenzgebiete, 68. Springer, Berlin etc., 1972. Zbl 0254.22005 MR 0507234

[22] T. A. Springer, Linear algebraic groups. $2^{\text {nd }}$ ed., Progress in Mathematics, 9. Birkhäuser Boston, Boston, MA, 1998. Zbl 0927.20024 MR 1642713

[23] V. S. Varadarajan, Lie groups, Lie algebras, and their representations. Reprint of the 1974 ed. Graduate Texts in Mathematics, 102. Springer-Verlag, New York, 1984. Zbl 0955.22500 MR 0746308

[24] F. W. Warner, Foundations of differentiable manifolds and Lie groups. Scott, Foresman and Co., Glenview, IL, and London, 1971. Zbl 0241.58001 MR 0295244 
Received February 20, 2012

Meera G. Mainkar, Department of Mathematics, Pearce Hall,

Central Michigan University, Mt. Pleasant, MI 48859, U.S.A.

e-mail: maink1m@cmich.edu 